\title{
Etnomatemática de una Artesanía Argentina: identificando etnomodelos de trenzado
}

\section{Ethnomathematics of an Argentine Craft: identifing ethnomodels of braid}

\author{
Veronica Albanese*
}

\begin{abstract}
Resumen
La investigación se propone caracterizar las matemáticas presentes en la labor artesanal soguera argentina de la realización de trenzas. A tal fin se acomete una interpretación matemática, situada mediante la modelización de la práctica del trenzado desde una perspectiva émica. Para ello, se parte de la reorganización del espacio a partir de las actividades matemáticas universales de localizar y diseñar y de una visión amplia de matemáticas como sistema que trata los aspectos cuantitativos relacionales y espaciales de la experiencia humana (sistema $Q R S)$. A través del concepto clave de etnomodelo se describen las formas en que los artesanos organizan el espacio y el sistema simbólico que constituye el lenguaje utilizado para comunicarse.
\end{abstract}

Palabras-Clave: Etnomatemáticas. Artesanías de Trenzado. Émico. Etnomodelo. Matemáticas como Sistemas QRS.

\begin{abstract}
This investigation focuses on the study of the mathematics of an Argentine craftsmanship of making braids. The objective is to develop a situated mathematical interpretation for the modeling of the braiding practice from an emic perspective. We set out from the reorganization of the space through the universal mathematical activities to locate and design and a broad view of mathematics as a system that deal with the relational, quantitative, and spatial aspects of human experience (QRS-system). Through the key concept of ethnomodels, we describe the ways in which artisans organize the space and we describe the symbolic system which is the language they use to communicate.
\end{abstract}

Keywords: Ethnomathematics. Braid Crafts. Emic. Ethnomodel. Mathematics as QRS Systems.

\section{Planteamiento}

La Etnomatemática se define, inicialmente, como el estudio de las prácticas matemáticas de grupos culturalmente determinados (D’AMBROSIO, 2008) para evolucionar como un programa que abarca una nueva concepción amplia del conocimiento como los

\footnotetext{
* Doctora en Educación por la Universidad de Granada (UGR). Investigadora y Profesora contratada por la Universidad de Granada (UGR), Granada, España. Dirección postal: Facultad de Educación, Departamento de Didáctica de las Ciencias Experimentales, Campus Cartuja 18071, Granada, España. E-mail: very_alba@hotmail.it.
} 
modos, estilos, artes y técnicas - Ticas - de explicar, aprender, conocer, relacionarse con Matema - el ambiente natural, social y cultural - Etno - (D’AMBROSIO, 2012).

El contexto de esta investigación es un gremio de artesanos de la provincia de Buenos Aires, Argentina, que se dedica a la realización de artefactos con cuero crudo. A esta artesanía se le proporcionan varios nombres, según la región; él que se usa en la provincia bonaerense es soguería gaucha criolla, porque uno de sus productos principales son las sogas que se utilizan para montar a caballo y porque es practicada originariamente por los gauchos criollos, hombres semi-nómadas y solitarios que se dedicaban a criar ganado en la zona pampeana.

En particular, en este documento nos centramos en la realización de trenzas con tientos de cuero. Los tientos son tiras cortadas de lonjas de cuero crudo de potro o chivo. Puntualizamos que por artesanía entendemos la labor de creación o decoración, de manera predominantemente manual y artística, de objetos de utilidad práctica en la sociedad. Las trenzas son productos de un tipo de tejido simple en las cuales predomina una dimensión y se realizan solo con las manos, es decir, sin el empleo de herramientas suplementarias (OLIVERAS; ALBANESE, 2012).

\subsection{Antecedentes}

Pionero en el estudio etnomatemático de las prácticas matemáticas de los gremios artesanales son el estudio de Millroy (1991) con carpinteros y el trabajo doctoral de Oliveras (1996) sobre artesanía andaluzas, de los cuales tomamos la secuenciación de la práctica artesanal en fases.

Del trabajo de Albertí (2007), que se propone identificar matemáticas en la labor de ornamentación arquitectónica del pueblo Toraja, de una isla de Indonesia, nos interesan las implicaciones de la distinción entre proyección matemática e interpretación matemática situada. La primera es una modelización o identificación de un concepto o razonamiento matemático que es visto e impuesto por el investigador en la práctica artesanal sin evidencias de reconocimiento del mismo por parte del artesano. La segunda, la interpretación matemática situada, se realiza cuando el investigador reconoce la presencia del concepto o razonamiento matemático en el pensamiento propio del artesano.

Rosa y Orey (2012) se enfrentan a esta misma distinción en términos antropológicos. Ellos identifican en la construcción de las casas del pueblo indígena norteamericano Sioux, la 
carpa cónica, dos tipos de interpretaciones, una ética y una émica ${ }^{2}$. La interpretación ética se basa en las categorías de análisis de la cultura del investigador, la émica tiene en cuenta las categorías propias de la cultura indígena estudiada. Los mismos autores introducen una tercera aproximación, la dialéctica, de forma tal que las perspectivas ética y emicá sean complementarias y no contrapuestas. Nuestro entendimiento de las dos perspectivas no se sitúa en la idea de contraposición de enfoques: creemos que ambas perspectivas, ética y émica, no pueden prescindir la una de la otra, así que en este trabajo no consideramos la dialéctica por estar esta ya incorporada en nuestra visión. Afirmar que nos posicionamos desde una perspectiva émica equivale, para nosotros, a una declaración de la aproximación inicial, del esfuerzo de la investigación por representar también - y sobretodo partir de - el punto de vista del grupo cultural estudiado. En nuestra postura asumimos y somos conscientes que cada forma de análisis posterior será influenciada por la cultura y lógica académica (perspectiva ética).

Con respecto al estudio de los tejidos, mencionamos algunas investigaciones que se interesaron en descubrir patrones en la elaboración de la trama: el trabajo Gavarrete (2012) sobre artesanía cestera de un pueblo originario de Costa Rica, el estudio de Parra (2003) sobre la fabricación de sogas por los indígenas Ticuna de la Amazonia colombiana y las investigaciones del grupo portugués de Palhares (2008).

El antecedente de referencia para nuestro estudio es la investigación sobre artesanía de trenzado de la región de Salta, que se plantea en Oliveras y Albanese (2012). La modelización conseguida a partir de una observación etnográfica de ese escenario, basada en la utilización de grafos (ALBANESE; OLIVERAS; PERALES, 2012), se podría aplicar, también, a la realización de las trenzas por parte de los artesanos sogueros (ALBANESE; OLIVERAS; PERALES, 2014). Sin embargo, surge la pregunta acerca de si dicha modelización pudiera constituir una proyección matemática, una imposición realizada por el investigador, en otras palabras una modelización ética. Para encontrar una respuesta es preciso averiguar cuál es la interpretación matemática situada, esto es, la forma propia de los artesanos de modelizar su propia práctica, la modelización émica.

\section{Fundamentos teóricos}

\footnotetext{
${ }^{2}$ Estos términos son prestados de la antropología cultural.
} 
Revisamos los planteamientos de algunos autores sobre la concepción de matemáticas, en particular en relación al espacio.

El educador matemático Allan Bishop (1999), sensible a la concepción de matemáticas como producto cultural, se propone identificar las universalidades presentes y comunes a todas las culturas y que se pudieran definir como matemáticas. Determina que las matemáticas son un "conjunto de conocimiento simbolizado resultante de determinadas actividades" (BISHOP, 1999, p.42), e indica algunas actividades matemáticas universales (AMU), o prácticas invariantes, como origen de todo desarrollo matemático. La idea de hablar de las actividades surge de su concepción del conocimiento como fruto de la relación del hombre con el entorno en el cual está inmerso. Contar y medir son actividades que, en muchas culturas, se desarrollan a partir del propio cuerpo, el entorno individual; localizar y diseñar son actividades que nacen del entorno espacial; jugar y explicar son actividades que surgen en las relaciones interpersonales, el entorno social.

En este trabajo nos enfrentamos principalmente con prácticas relacionadas con el espacio y, por consiguiente, trataremos las actividades de localizar y diseñar (BISHOP, 1999).

La actividad matemática universal de diseñar se refiere a los artefactos que toda cultura crea para su vida cotidiana:

\begin{abstract}
La esencia de diseñar es transformar una parte de la naturaleza, es decir, tomar un fenómeno natural, sea madera, arcilla o terreno y transformarlo en otra cosa (...). Diseñar implicar imponer una estructura particular a la naturaleza. (...) Diseñar implica imaginar la naturaleza sin las partes innecesarias y quizás incluso destacar algunos aspectos por encima de otros. Así pues, diseñar consiste en gran medida, en abstraer una forma del entorno natural. Lo que es importante para nosotros en educación matemática es el plan, la estructura, la forma imaginada, la relación espacial percibida entre objeto y propósito, la forma abstracta y el proceso de abstracción (BISHOP, 1999, p. 61).
\end{abstract}

Diseñar es una manera de modelizar para interpretar el entorno, con el objetivo de entenderlo, modificarlo o recrearlo, dándole forma. Es, quizás, la actividad que tiene la conexión más inmediata con el entorno (BISHOP, 1999, p.135).

La actividad universal de localizar se refiere a la situación de uno mismo y de otros objetos en el entorno espacial. "Localizar enfatiza la geometría espacial de la posición y del movimiento" (BISHOP, 1999, p.133).

A propósito del entorno espacial, el antropólogo cultural Rix Pinxten (1987), para el desarrollo del UFOR (Universal Frame of Reference) - un instrumento analítico que permite estudiar las nociones espaciales en contextos culturales diferentes y que utiliza para exponer la filosofía y la fenomenología del espacio de la tribu de los Navajos - concibe tres 
dimensiones: 1) El espacio físico o de objetos que abarca todas esas características del espacio o fenómenos espaciales que el hombre puede manipular, manejar, considerar y desplazar en su totalidad, actuando sobre estos en su totalidad, o sea, fenómenos relativamente pequeños. 2) El espacio socio geográfico que está constituido por los fenómenos espaciales con los cuales el hombre puede ser confrontado o dentro el cual el hombre puede situarse, sin poder manipularlo (ej. una casa, un país). 3) El espacio cosmológico que está compuesto por fenómenos de magnitud todavía mayor (ej. el Universo, la Tierra, los fenómenos celestes), el hombre tiene experiencia de ellos de manera indirecta u observándolos a distancia. Si bien Bishop centra sus reflexiones sobre el espacio socio-geográfico, aquí consideramos el espacio físico o de objetos, ya que el contexto de la investigación es una artesanía. En esta dimensión el espacio está generalmente orientado en relación a la posición de la persona que lo maneja.

Bishop (1999) destaca que la actividad de localizar desarrolla el lenguaje y los símbolos para describir localizaciones e incluye los procedimientos de reducción a escala del entorno (ej. mapas).

Las actividades de Bishop de diseñar y localizar sugieren, entonces, el desarrollo de dos tipos de representaciones que indicamos respectivamente como representación icónica, un dibujo o una imagen mental, y representación simbólica, como el lenguaje (BRUNER, 1984; 1988). Una "representación, o un sistema de representaciones es un conjunto de reglas mediante las cuales se puede conservar aquello experimentado en diferentes acontecimientos" (BRUNER, 1984, p.122).

Barton (2008) propone concebir las matemáticas como las maneras de entender los aspectos cuantitativos, espaciales y relacionales de la realidad; es el lenguaje que se utiliza para representar y entender estos aspectos, tanto el que se emplea en los libros de textos como el que pueda usar un artesano.

Compartimos, entonces, la visión que Barton llega a plantear de las matemáticas como sistema QRS, es decir "a system for dealing with quantitative, relational, or spatial aspects of human experience" (BARTON, 2008, p.10).

Finalmente, Rosa y Orey (2012) definen el concepto de etnomodelo que será clave para nosotros. Los etnomodelos son modelos culturales, herramientas de comprensión del saber, en nuestro caso, del gremio artesanal que son representaciones externas y consistentes con el conocimiento matemático que es construido y compartido socialmente por el grupo de artesanos. 
Organizamos las aportaciones de estos según la Figura 1, y puntualizamos que en el análisis utilizaremos fundamentalmente los conceptos que enmarcamos en la circunferencia discontinua.

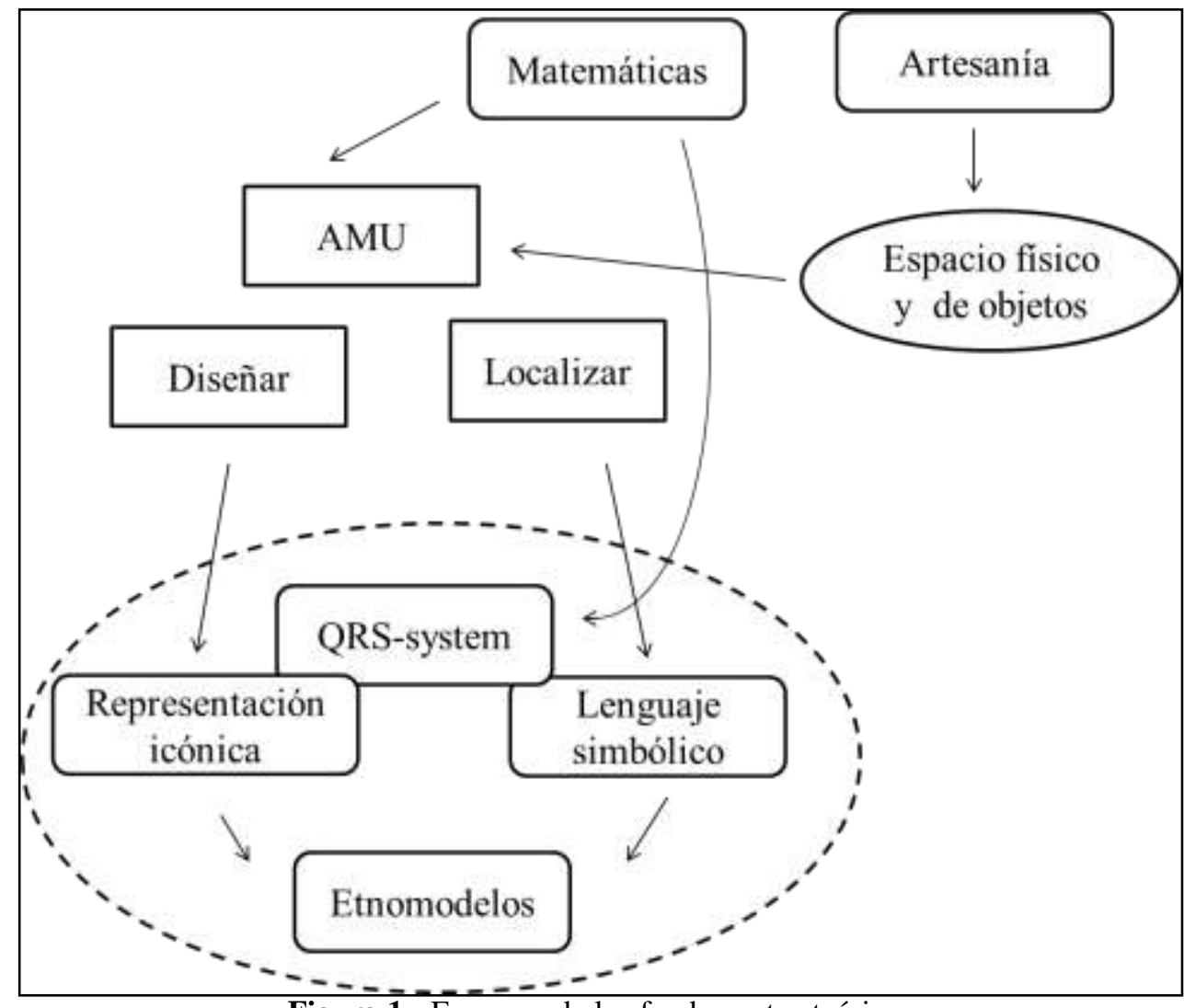

Figura 1 - Esquema de los fundamentos teóricos

\section{Objetivos y metodología}

El objetivo de la investigación es caracterizar las matemáticas que subyacen en la labor artesanal soguera de la realización de trenzas. Queremos describir el sistema al cual recurren los artesanos para tratar los aspectos principalmente espaciales de su práctica. Apuntamos a describir sus sistemas de representaciones del espacio, los etnomodelos, a través de elementos icónicos y simbólicos, en el intento de conocer y entender la manera de organizar el trabajo y las matemáticas implicadas en esta tarea.

El propósito de la investigación se concreta en comprender las maneras de ver, entender y representar matemáticamente la práctica, según la visión de los artesanos desde una perspectiva émica (ROSA; OREY, 2012), con las matices descritas en la sección 1.1. Para vislumbrar el punto de vista de los artesanos y sus formas de representar el proceso de realización del trabajo artesanal, la investigación se inserta en el marco del interaccionismo simbólico, es decir, consideramos que los significados se construyen y se comparten cuando 
los individuos interactúan (ANGROSINO, 2012), y estas interacciones tienen que ser observadas en su entorno natural.

Por esta razón se ha optado por una metodología etnográfica que implica una inmersión en el campo con observación participante (ANGROSINO, 2012), para vivir en primera persona un proceso de enculturación y llegar a penetrar en las formas de pensar propias de la artesanía considerada.

En el 2012, en la ciudad de Buenos Aires, la investigadora ha sido aprendiz en dos cursos de soguería durante 10 semanas. Ambos cursos eran impartidos en forma de taller. Los tres artesanos (en un curso dos artesanos, A y B, daban clase de manera conjunta) conocían los propósitos de la investigación y firmaron un consentimiento informado. Los datos recolectados durante la observación participante forman parte del diario de campo de la investigadora-aprendiz, así como las fotos de los trabajos realizados en las clases, los apuntes y fotocopias proporcionados por los artesanos como material didáctico. Al final de la inmersión en el escenario se han realizado unas entrevistas etnográficas que han sido grabadas en audio y transcritas.

El análisis de datos cualitativos de tipo descriptivo-interpretativo se ha realizado con la finalidad de comprender e interpretar la realidad tal y como es entendida por los artesanos. Con el apoyo del programa informático MAXQDA7, se han organizado, manipulado y recuperado los segmentos significativos de los datos, comenzando con la identificación de temas o patrones-clave para, después, definir categorías asignándoles códigos (COFFREY; ATKINSON, 2005).

\subsection{Trabajo de campo etnográfico}

A continuación relatamos el desarrollo del trabajo de campo etnográfico que resumimos en el Cuadro 1.

\begin{tabular}{|l|l|}
\hline Periodo & Fase del trabajo de campo \\
\hline Semanas 1 y 2 & Entrada en el campo y vagabundeo \\
\hline Semanas 3, 4, y 5 & Familiarización con el lenguaje técnico \\
\hline Semanas 6 y 7 & Consolidación y afianzamiento de la práctica y conocimiento artesanal \\
\hline Semanas 8,9 & Revisión de los datos; hipótesis con identificación de etnomodelos \\
\hline Semana 10 & Entrevistas: refinamiento y "validez comunal" de los etnomodelos \\
\hline
\end{tabular}

Cuadro 1 - Fases del trabajo de campo etnográfico

En las dos primeras semanas se realiza la entrada en el campo: la investigadoraaprendiz contacta con los artesanos, les comunica los propósitos de la investigación y comienzan las clases de soguería. De esta primera fase de vagabundeo (ANGROSINO, 2012) 
cabe destacar tres acontecimientos: 1) se contacta con un cuarto artesano, cuya escasa empatía con el estudio hizo que descartásemos su participación en la investigación, además de no ser muy conocido en el gremio; 2) a través de los materiales que conseguimos en una de las más importantes ferias anuales (La Rural) donde se presentan trabajos de soguería, principalmente aportaciones a la publicación de una revista especializada, y de charlas informármeles con artesanos allí presentes, tuvimos la confirma de la importancia en el gremio de los artesanos cuyos cursos elegimos seguir; 3) decidimos incluir como objetos de investigación otros artefactos cuyos análisis se presentan en otra publicación (ALBANESE; PERALES, 2014).

En las sucesivas tres semanas $(3,4,5)$ la investigadora-aprendiz empieza a adquirir cierta familiaridad con el lenguaje utilizado por los artesanos e identifica ciertas palabras recurrentes en sus discursos; además, aprende a interpretar los dibujos y a realizar trenzas simples, al principio con cierta dificultad cuando el número de tientos es relativamente grande. Durante las dos semanas siguientes $(6,7)$ la investigadora-aprendiz consolida su habilidad y conocimiento de la práctica de trenzar y de la comunicación sobre esta; además, adquiere confianza con el lenguaje simbólico escrito y razona sobre patrones simples que se encuentran en la estructura de tal lenguaje. En la semanas 8 y 9 a través de la reflexividad (ANGROSINO, 2012) en la revisión de los datos, y entonces de una fase de pre-análisis, surge la hipótesis sobre la identificación de los etnomodelos que se describen en detalle más adelante (Sección 4.1). Esto proporciona a la investigadora-aprendiz un acceso más ágil a las informaciones y a los manuales y publicaciones de la comunidad artesanal sin, en la mayoría de las veces, necesidad de la mediación de los artesanos para la realización de trenzas con un elevado número de tientos.

En la última semana se realizan las entrevistas, estas permiten refinar la formulación de los etnomodelos e, implícitamente, se obtiene una validez comunal, es decir los artesanos avalan y confirman, cada uno en su medida, el utilizo y la preferencia de unos etnomodelos sobre otros (véanse la sección 4.2).

\section{Resultados del análisis interpretativo}

Presentamos algunos resultados del análisis cualitativo de los datos, en particular consideramos:

- Las fases naturales de la labor artesanal. 
- Los etnomodelos que identificamos en las explicaciones de los artesanos, es decir, los sistemas QRS (BARTON, 2008) que los artesanos desarrollan y utilizan para organizar su trabajo y comunicarse sobre ellos.

- Las relaciones entre los etnomodelos y los tres artesanos.

Desde la primera observación pudimos distinguir dos fases en el proceso de realización de las trenzas. La primera caracteriza el principio, cómo se van disponiendo los tientos cuando se empieza el trabajo, la disposición inicial. La fase sucesiva es la del trenzado propiamente, que consiste en ir tejiendo la trama de la trenza según unos patrones establecidos y de forma reiterativa.

\subsection{Los etnomodelos}

Los etnomodelos (ROSA; OREY, 2012) emergieron del proceso de aprendizaje de la investigadora-aprendiz. Inicialmente, encontramos dos etnomodelos empleados por los artesanos y en un segundo momento, cuando ya se puso la atención en las actuaciones de los artesanos y se realizaron las entrevistas, se confirmó la existencia de un tercero (Cuadro 2).

\begin{tabular}{|l|l|}
\hline \multicolumn{1}{|c|}{ Etnomodelos } & \multicolumn{1}{|c|}{ Descripción } \\
\hline Etnomodelo D (dibujo) & $\begin{array}{l}\text { Dibujo, o serie de dibujos, que representa los tientos que se van } \\
\text { trenzando. }\end{array}$ \\
\hline Etnomodelo SB (Sobre/Bajo) & $\begin{array}{l}\text { Secuencia de pasadas que describen el proceso de trenzado: la mano que } \\
\text { trenza (I o D) y el recorrido del tiento, respecto a los otros tientos, (p. ej. } \\
\text { S2 B1). }\end{array}$ \\
\hline Etnomodelos R (Reglas) & $\begin{array}{l}\text { Las múltiples reglas, o patrones matemáticos, que rigen el etnomodelo } \\
\text { SB. Permiten inventar nueva trenzas, reconocer si las pasadas describen } \\
\text { una trenza que salga bien, o que termina saliendo igual que otra. }\end{array}$ \\
\hline
\end{tabular}

Cuadro 2 - Descripción de los etnomodelos

Etnomodelo D (D de Dibujo): aquí son protagonistas las representaciones icónicas ya que un dibujo refigura los tientos a punto de ser trenzados. Los tientos se dividen en dos subconjuntos, uno a la derecha (las rectas de los tientos tienen pendiente positiva /) y uno a la izquierda (las rectas con pendiente negativa \) según la mano que los sujeta (Figura 2). El proceso se representa con una secuencia de dibujos que indican los momentos sucesivos del trenzado. Los elementos simbólicos que pueden acompañar los dibujos son las etiquetas de los tientos con letras o números. Se suele recurrir a este etnomodelo generalmente para la fase de la disposición inicial y cuando se explica a aprendices principiantes. 


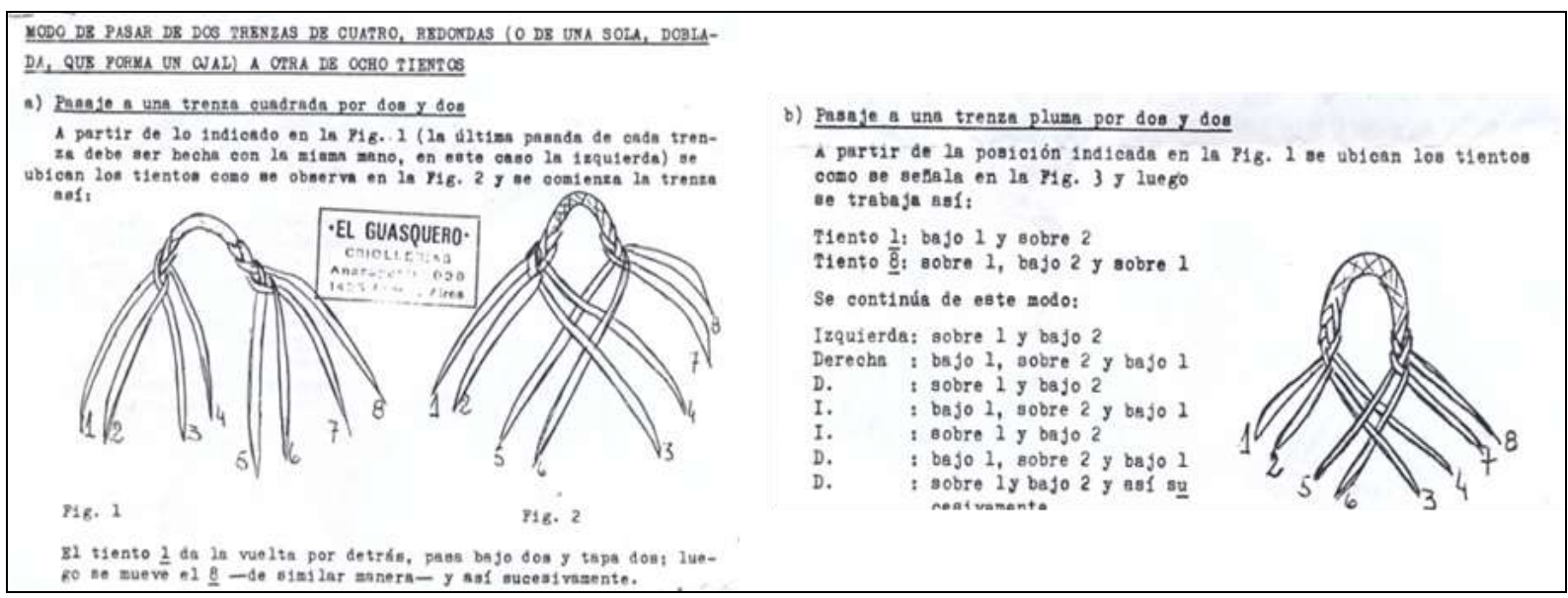

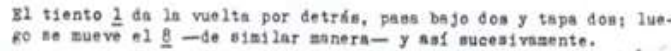

Figura 2 - Material proporcionado por los artesanos A y B con dibujos -etnomodelo D- de trenzas cuadrada (a) y chata (b); en esta última se observa también el etnomodelo SB con la descripción de las pasadas

Etnomodelo SB (SB de Sobre/Bajo): aquí prevalece el lenguaje simbólico (BRUNER, 1984, 1988), se basa en la convención implícito-tácita de que se trenza el tiento externo del lado de la mano que trabaja. La mano que trabaja se indica con I de Izquierda y D de Derecha. Un tiento externo trabaja una pasada hasta llegar a su nueva posición, después se pasa a otro tiento externo, el de la otra mano -para las trenzas más comunes (Cuadro 3)-, o el nuevo externo del mismo lado - para las trenzas patrias, donde se trabaja dos veces con la misma mano (Figura 3). La pasada siempre es hacia el centro, partiendo del mismo extremo del tiento que trabaja, cuando la trenza es chata (se dice impropiamente por delante), mientras si la trenza es redonda o cuadrada se pasa por detrás y se empieza a contar los tientos de la pasada por el extremo opuesto. En particular, si la pasada es, por ejemplo S2 B1, esto significa sobre (S) dos tientos, cubriéndolos a la vista de quien trenza, y bajo (B) un tiento, escondiendo el tiento que trabaja por debajo, con respecto a quien trenza, contando siempre desde el extremo hacia el centro.

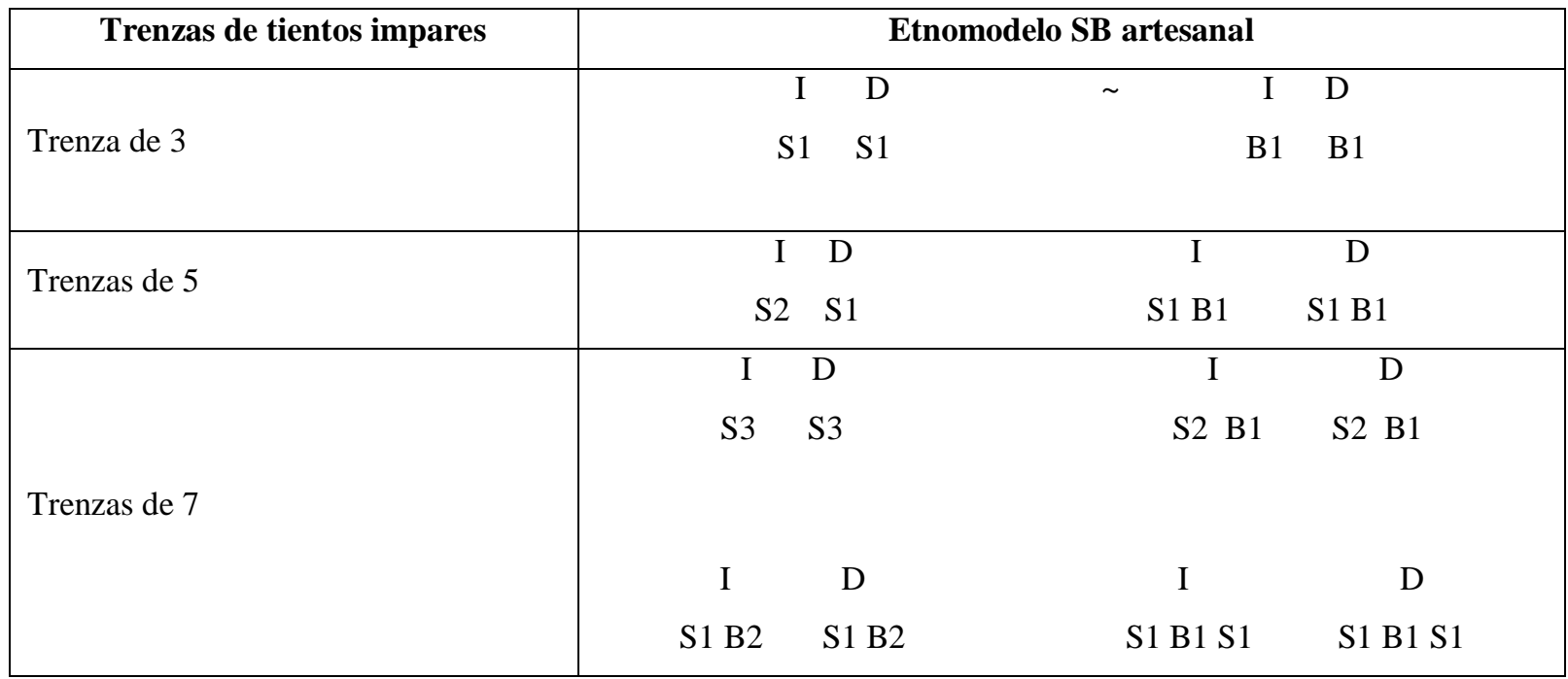

Cuadro 3 - Ejemplificativo del etnomodelo SB para trenzas de 3, 5 y 7 tientos 
En 1 cuadro 4, cada bloque compuesto por I y D con abajo el detalle de las pasadas describe una trenza. Las dos trenzas de 3 son equivalente, es decir que el producto final del trenzado es el mismo, mientras las otras trenzas de 5 y 7 son todas distintas. Para interpretar este lenguaje se parte de la convención que el tiento que trabaja es el externo del lado indicado por la letra de arriba (D por derecha, I por izquierda) y este se mueve hacia el centro siguiendo las instrucciones de la segunda línea, pasando S sobre (S), o bajo (B), el número de tientos indicados después de la $\mathrm{S}$ o $\mathrm{B}$.

A veces S y B se sustituyen con los símbolos más + y menos - (Figura 3). Se suele utilizar este etnomodelo para la fase del trenzado propiamente dicho, cuando se manejan un número de tientos elevado (más de 4 o 6) y cuando se supone que los interlocutores ya tienen cierto manejo de la labor de trenzar.

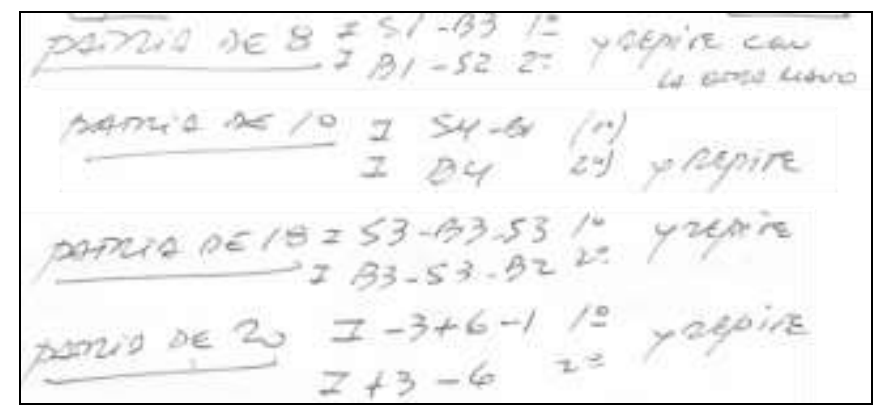

Figura 3 - Material proporcionado por el artesano B con pasadas de trenzas patrias

Consideramos que en este etnomodelo SB se desarrolla un sistema QRS (BARTON, 2008) propio que el gremio artesanal acepta y maneja para describir las trenzas y es compartido por los artesanos porque lo pudimos observar también en algunas publicaciones propias de la comunidad artesanal soguera (OSORNIO, 1934).

Etnomodelos R (R de Reglas): estos etnomodelos abarcan las observaciones de los artesanos acerca de reglas lógicas que rigen los patrones numéricos que se repiten en el trenzado, o las relaciones que se establecen entre el número de tientos y las trenzas posibles con esa cantidad de tientos. Podemos considerar estos etnomodelos como de un nivel superior o más complejo, ya que para manejarlos se necesita llegar a una comprensión profunda del lenguaje del etnomodelo SB y se necesita cierto grado de abstracción de tipo matemático combinatorio.

Proporcionamos algunos ejemplos que comentaron los artesanos.

- El reconocimiento de los patrones que rigen el lenguaje artesanal permite inventar trenzas útiles ya que toda trenza respeta unas pautas de simetría, en las que tienen un número de tientos impar las pasadas de ambas manos son iguales. Esto se debe a que una trenza que 
no respeta esas pautas - según los artesanos - es fea y no sirve, porque es más débil, se rompe, ya que los tientos se tensan de manera distinta.

- $\mathrm{Al}$ aumentar el número de tientos aumentan las posibilidades de realizar diferentes trenzas y esto involucra un pensamiento combinatorio. Sin embargo, algunas trenzas terminan siendo iguales, como dos trenzas de 3 en la primera fila del Cuadro 3.

- Hay relaciones numéricas entre el número de tientos y la suma de los tientos que se involucran en una pasada según el tipo de trenza (chata, redonda o patria), además estas relaciones son muy diferentes si el número de tientos con los cuales se trabaja es par o impar. En particular, con un número $\mathrm{n}$ impar de tientos las pasadas de las trenzas chatas suelen involucrar (n-1)/2 tientos (Cuadro 3).

\subsection{Relación entre etnomodelos y artesanos}

Finalmente, observamos la relación de los etnomodelos con los artesanos (Figura 4).

\begin{tabular}{|l|l|l|l|}
\hline Ystem & ma A & ma B & ma C \\
\hline Etnomodelo trenza & & & \\
틀 D-Dibujo & & \\
틀 SB-Sobre Bajo & & \\
틀 R-reglas & & \\
\hline
\end{tabular}

Figura 4 - Matriz de códigos generada por MAXQDA7 entre los artesanos y los etnomodelos. La magnitud de los cuadrados indica la frecuencia relativa de los códigos

En el curso de los artesanos A y B se consiguieron mucha información sobre las trenzas. En particular, el artesano B proporcionó muchas explicaciones, al principio con material que contenía dibujos (Figura 2), y, una vez que la investigadora-aprendiz se había familiarizado con la técnica y el manejo del lenguaje simbólico o etnomodelo SB, acudía a esquemas en donde proporcionaba las pasadas, como se observa en la Figura 3. Se observó que durante la construcción del esquema el artesano realizaba varios comentarios sobre los patrones que mencionamos como perteneciente a los etnomodelos $\mathrm{R}$.

El artesano A facilitó información sobre las trenzas durante la entrevista que se realizó al final de la inmersión en el entorno artesanal. Apuntó, explícitamente, a las reglas lógicas que consideramos como etnomodelos R. Además, cabe destacar que hizo referencia a las formas de aprender la labor artesanal y generar nuevas trenzas que se manejan en ese contexto: el ensayo-error, la observación del producto finito - si hay un ejemplar a copiar -, las similitudes lógicas entre los trenzados para aprender y los ya conocidos (etnomodelos R).

El artesano C fomenta el aprendizaje por imitación y por descubrimiento. Él suele mostrar cómo se realiza la trenza sin proporcionar explicaciones verbales, para que cada 
aprendiz pueda observar los patrones y desarrollar sus propias técnicas de elaborar y recordar. Menciona las pasadas propias del etnomodelo SB y, durante la entrevista, manifiesta un patrón lógico de etnomodelo $\mathrm{R}$ relacionado con la producción de otros artefactos de esta misma artesanía (ALBANESE; PERALES, 2014).

\section{Reflexiones finales}

Cabe destacar la contribución del trabajo a las investigaciones etnomatemáticas por el uso de etnomodelos como herramienta para la descripción de prácticas artesanales.

La investigación responde a un interrogante dejado abierto en una investigación anterior (ALBANESE; OLIVERAS; PERALES, 2014) sobre cuál es la interpretación matemática situada por parte de los artesanos sogueros respecto a la modelización de su propia práctica de trenzado desde la perspectiva émica. Con el desarrollo de los etnomodelos se describen las formas en que los artesanos organizan el espacio, además de cómo piensan y comunican su trabajo, y se descarta la utilización de grafos, como se planteó en Oliveras y Albanese (2012).

En la determinación de los etnomodelos destacamos la importancia que adquiere el lenguaje, concretamente las expresiones orales y el sistema simbólico escrito, para la comunicación entre los miembros de la comunidad artesanal sobre la realización del trenzado. En este sentido, estos etnomodelos son parte de un sistema QRS que desarrollan los artesanos para organizar y compartir la práctica. Asimismo, hemos constatado que no todos los artesanos proporcionan evidencias del uso de los mismos etnomodelos, aunque el etnomodelo SB parece consensuado dentro de la comunidad por las evidencias que hemos mencionado.

Ponemos de manifiesto que el desarrollo de un etnomodelo implica un consenso sobre unos supuestos básicos, a menudo implícitos, a partir de los cuales se construye el lenguaje y el sistema simbólico y ¿qué son los axiomas sino el equivalente en las matemáticas formales de estos supuestos básicos?

A nivel educativo, manifestamos las potencialidades de la introducción de actividades curriculares basadas en las Etnomatemáticas, como las presentadas en Albanese, Oliveras y Perales (2012) y en metodologías investigativas de búsqueda de matemáticas en contextos de la vida real, con especial atención a la distinción e integración entre los puntos de vistas ético y émico, en particular para la formación docente y con el propósito de incidir en las concepciones epistemológicas sobre la naturaleza de las matemáticas (ALBANESE; SANTILLÁN; OLIVERAS, 2014). 


\section{Agradecimientos}

Expresamos nuestro profundo agradecimiento a los artesanos que pusieron a disposición sus conocimientos y su tiempo para la realización de esta investigación.

Agradecemos el Ministerio de Educación, Cultura y Deporte del Gobierno de España, que soporta esta investigación con una Beca FPU (código de referencia AP2010-0235).

\section{Referencias}

ALBANESE, V.; PERALES, F. J. Pensar Matemáticamente: Una Visión Etnomatemática de la Práctica Artesanal Soguera. RELIME - Revista latinoamericana de investigación en matemática educativa, Mexico DF, v. 17, n. 3, p. 261-288, Nov. 2014.

ALBANESE, V.; SANTILLÁN, A.; OLIVERAS, M. L. Etnomatemática y formación docente: el contexto argentino. Revista Latinoamericana de Etnomatemática, San Juan de Pasto (Colombia), v. 7, n. 1, p. 198-220, Abr. 2014.

ALBANESE, V.; OLIVERAS, M. L.; PERALES, F. Etnomatemáticas en Artesanías de Trenzado: Aplicación de un Modelo Metodológico elaborado. BOLEMA - Boletim de Educação Matemática, Rio Claro (São Paulo), v. 28, n. 48, p. 1-20, Abr. 2014.

ALBANESE, V.; OLIVERAS, M. L.; PERALES, F. Modelización matemática del trenzado artesanal. Revista Épsilon, Córdoba (España), v. 29, n. 81, p. 53-62, Dic. 2012.

ALBERTÍ, M. Interpretación matemática situada de una práctica artesanal. 2007. 379 f. Tesis (Doctorado en Didáctica de la Matemática y de la Ciencias Experimentales) - Facultad de Educación, Universidad Autónoma de Barcelona, Barcelona, 2007.

ANGROSINO, M. Etnografía y observación participante en Investigación Cualitativa. Barcelona: Morata, 2012.

BARTON, B. The language of mathematics: Telling mathematical tales. Melbourne: Springer, 2008.

BISHOP, A. J. Enculturación Matemática. Barcelona: Paidós, 1999.

BRUNER, J. S. Acción, pensamiento y lenguaje. Madrid: Alianza Psicología, 1984.

BRUNER, J. S. Desarrollo cognitivo y educación. Madrid: Morata, 1988.

COFFREY, A.; ATKINSON, P. Encontrar el sentido a los datos cualitativos: Estrategia complementaria de investigación. Alicante: Editorial Universidad de Alicante, 2005.

D'AMBROSIO, U. The Program Ethnomathematics: theoretical basis and the dynamics of cultural encounters. Cosmopolis, Bousval (Bélgica), n. 3-4, p. 13-41, Oct. 2012.

D'AMBROSIO, U. Etnomatemática: Eslabón entre las tradiciones y la modernidad. México: Limusa, 2008.

GAVARReTe, M. Matemáticas, Culturas y Formación de Profesores en Costa Rica. 2013. 734 f. Tesis (Doctorado en Ciencia de la Educación) - Departamento de Didáctica de la Matemática, Facultad de Educación, Universidad de Granada, Granada, 2012. 
MILLROY, W. L. An ethnographic study of the mathematical ideas of a group of carpenters. Learning and Individual Differences, New Haven (Connecticut), v. 3, n. 1, p. 1-25, Feb. 1991.

OLIVERAS, M. L.; ALBANESE, V. Etnomatemáticas en Artesanías de Trenzado: Un Modelo Metodológico para Investigación. BOLEMA - Boletim de Educação Matemática, Rio Claro (São Paulo), v. 26, n. 44, p. 1295-1324, Dic. 2012.

OLIVERAS, M. L. Etnomatemáticas. Formación de profesores e innovación curricular. Granada: Comares, 1996.

OSORNIO, M. Trenzas gauchas. Buenos Aires: Hemisferio Sur, 1934.

PALHARES, P. Etnomátematica. Um Olhar sobre a Diversidade Cultural e a Aprendizegem Matemática. Ribeirão (Portugal): Ediçoes Humus, 2008.

PARRA, A. Acercamiento a la Etnomatemática. 2003. 156 f. Tesis (Licenciatura en Matemática) Departamento de Matemáticas, Universidad Nacional de Colombia, Bogotá, 2003.

PINXTEN, R. Navajo Indian Geometry. Ghent: Commuication and cognition, 1987.

ROSA, M.; OREY, D. C. The field of research in ethnomodeling: emic, ethical and dialectical approaches. Educação e Pesquisa, São Paulo, v. 38, n. 4, p. 865-879, Oct. 2012.

Submetido em Abril de 2014. Aprovado em Março de 2015. 